Rev. Elev. Mod. vét. Pays trop., 1975, 28 (4) : 523-545

\title{
La race ovine Djallonké au Cameroun Potentialités zootechniques, conditions d'élevage, avenir
}

\author{
par F. VALLERAND (*) et R, BRANCKAERT $\left(^{* *}\right)$
}

\begin{abstract}
RESUME
Les auteurs ont poursuivi pendant neuf ans l'étude et la sélection du mouton Diallonké à la station de Nkolbisson située à $10 \mathrm{~km}$ de Yaoundé (Cameroun) et à travers de multiples enquêtes dans de nombreuses régions de l'Ouest et du Centre Cameroun. Les informations et résultats obtenus, tant sur les plans zootechnique et vétérinaire que dans le domaine socioéconomique, laissent entrevoir les grandes possibilités d'amélioration recélées par cette race. Cependant, compte tenu des conditions économiques actuelles, l'élevage intensif du mouton Djallonké ne s'avère guère rentable. Par ailleurs, il conviendrait d'améliorer le potentiel laitier des brebis et ceci pourrait, à l'avenir, être réalisé par l'infusion de sang exotique provenant d'une race prolifique et laitière, telle la race Romanov.
\end{abstract}

\section{INTRODUCTION}

Sur toute la Côte occidentale d'Afrique, de la Guinée à l'Angola, on trouve un mouton de petit format que Doutressoulle (2) appelle la race Djallonké. Selon cet auteur, elle serait originaire du Fouta-Djallon.

La bibliographie sur cette race est assez sommaire. A notre connaissance, seul le Centre de Recherches Zootechniques de Sotuba (10) en a étudié quelques paramètres zootechniques. La viande de mouton étant appréciée par les africains et plus particulièrement par les musulmans, il apparaît possible de développer cette spéculation qui, bien que pratiquée de façon empirique et non commerciale, représente pour le seul territoire de la République Unie du Cameroun un effectif d'environ 1,5 million de

(*) Département de Zootechnie, Ecole nationale supérieure agronomique, Université de Yaoundé, Cameroun.

(**) Senior Scientist Officer, Centre International pour l'Elevage en Afrique, B.P. 5689, Addis-Abeba, Ethiopie. têtes et une recette estimée à 800 millions de F CFA par an (1).

Comme nous le verrons, l'intensification de cette spéculation suppose une conversion complète des mentalités, singulièrement dans la zone forestière, mais avant de proposer à la vulgarisation des techniques appropriées, voire de nouvelles races, il est indispensable de connaître les paramètres zootechniques moyens de l'élevage en race pure de ce mouton. Nous nous sommes attelés à cette tâche d'évaluation depuis 1965 et nous proposons ici les résultats de ces recherches.

\section{DESCRIPTION DE LA RACE DJALLONKE}

L'allure générale du mouton Djallonké est sensiblement la même suivant les régions du Cameroun et les photos 1 et 2 en donnent un aperçu. Suivant les régions, on rencontre des variations de format dues probablement aux conditions de vie. Le mouton de forêt est généralement plus petit (25 kg pour les femelles) 

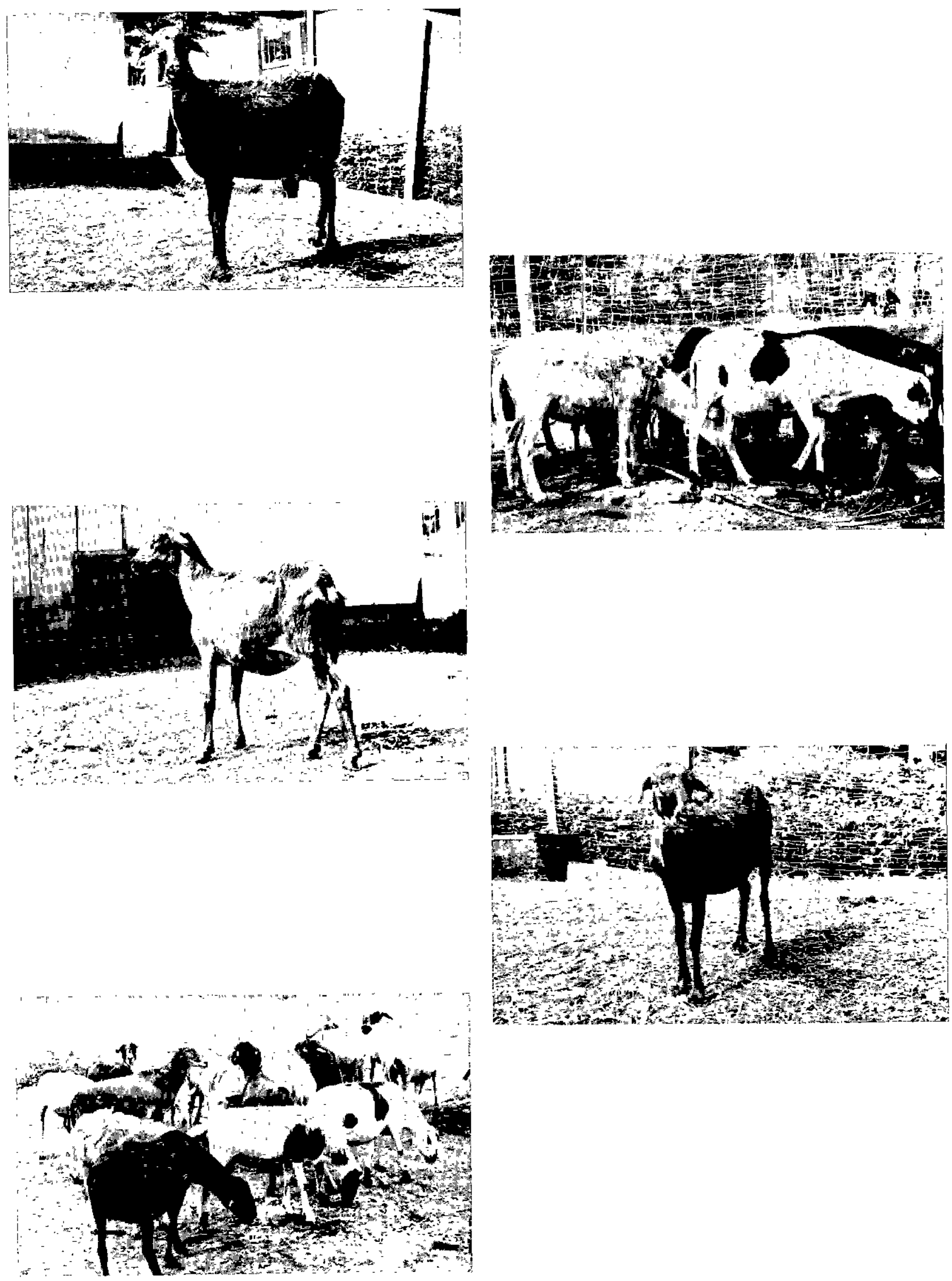
que le mouton des plateaux (1 000 à $2000 \mathrm{~m}$ ) de l'Ouest du pays $(30 \mathrm{~kg}$ ).

On pourra se faire une idée du format d'après les mensurations suivantes de brebis adultes de $25 \mathrm{~kg}$ en moyenne (3).

$\begin{array}{lll}\text { Périmètre thoracique } & 67 & \mathrm{~cm} \\ \text { Taille au garrot } & 59 & \mathrm{~cm} \\ \text { Largeur de poitrine } & 13,5 \mathrm{~cm} \\ \text { Profondeur de poitrine } & 25 \mathrm{~cm} \\ \text { Largeur coxo-fémorale } & 13 \mathrm{~cm}\end{array}$

Cet animal est donc assez haut sur pattes, peu éclaté et présente un développement musculaire faible des membres postérieurs.

Les robes les plus couramment rencontrées sont de couleur noire, pie-noire et plus rarement blanche, rouge, pie-rouge. Dans certaines régions (Est du Cameroun en particulier), on rencontre des animaux à robe particulière fauve avec le ventre et pattes noires. Cette sous-race est génétiquement stable et les animaux ont un aspect extérieur plus avantageux. Cette impression est d'ailleurs confirmée par leur poids moyen qui, chez les femelles adultes de cette sous-race, dépasse les $28 \mathrm{~kg}$.

Dans le nord du Cameroun on rencontre presque exclusivement les robes pie-noire et blanches. Ces moutons sont d'un format plus élevé et plus osseux, environ $75 \mathrm{~cm}$ au garrot. Ils proviennent probablement de croisements entre la race Djallonké et le mouton du Sahel couramment rencontré au Tchad. De plus en plus, nous assistons à un remplacement de ces « moutons du Nord » par la race Djallonké plus compacte et plus rustique semble-t-il, à tel point que dans la région, on distingue le " grand mouton du Tchad ou mouton du Ramadan » et le «petit mouton de Maroua».

La présence de cornes chez le mâle est loin d'être la règle, environ 50 p. 100 des mâles en sont porteurs. Quelques femelles portent des embryons de cornes. On trouve des pendeloques chez environ 5 p. 100 des moutons.

\section{SITUATION DE L'ELEVAGE OVIN AU CAMEROUN}

Le Cameroun peut être divisé en trois grandes régions climatiques :

- le Nord, sahélo-soudanien, zone fortement islamisée et où l'élevage est une activité traditionnelle ( 1 million de bovins). Pour des raisons sanitaires, cette région n'a pas de contact avec les autres régions d'élevage du Cameroun mais plutôt avec le Tchad et le Nigeria;

- le Centre, soudano-guinéen d'altitude $(1000 \mathrm{~m})$, est la zone de prédilection de l'élevage bovin ( 950000 têtes); région islamisée en partie mais sur laquelle on dispose de peu de données concernant les autres élevages;

- le Sud, guinéen, urbanisé à 27 p. 100, domaine des forêts et des plateaux (1 000 à $2000 \mathrm{~m}$ ), région des grandes cultures vivrières et d'exportation (café, cacao, bananes, palmiers...).

Une enquête récente de la SEDES (1) donne pour ces régions les effectifs, densités et consommation de viande de mouton résumés dans le tableau $\mathrm{n}^{\circ} \mathbf{I}$ ci-dessous.

Ce tableau récapitulatif appelle les commentaires suivants :

- l'implantation de l'Islam est un facteur non négligeable pour le développement de l'élevage ovin en corrélation avec la consommation de viande de mouton lors des fêtes religieuses musulmanes : fin du ramadan, fête du mouton. Si cette tendance n'est pas très nette pour le Centre, la part de viande de mouton dans la consommation toutes viandes réunies de la région Nord nous démontre l'intérêt porté par les populations islamisées à cet élevage. Dans ces deux régionș, la race élevée est : «]e mouton du Nord $»$ bien que le Centre soit de plus en plus implanté en ovins de race Djallonké proprement dite. Le mode d'élevage est distribué en deux tendances à peu près également représentées: le mouton de saré (ensemble de

TABLEAU N $N^{\circ}$ I

\begin{tabular}{|l|c|c|c|c|c|}
\hline Régions & $\begin{array}{c}\text { Effectif } \\
\text { ovins }\end{array}$ & $\begin{array}{c}\text { Densité } \\
\text { ovins/habitant }\end{array}$ & $\begin{array}{c}\text { Consommation de } \\
\text { viande de mouton/ } \\
\text { habitant/an }\end{array}$ & $\begin{array}{c}\text { Viande mouton } \\
\text { p.100 viandes } \\
\text { totaux }\end{array}$ & $\begin{array}{l}\text { Consommation toutes } \\
\text { viandes/habitant/an }\end{array}$ \\
\hline Nord & 700000 & 0,50 & $3,1 \mathrm{~kg}$ & 15 & $20,6 \mathrm{~kg}$ \\
Centre & 100000 & 0,12 & $0,8 \mathrm{~kg}$ & $?$ & $10,0 \mathrm{~kg}$ \\
\hline Sud & 450000 & 0,15 & $0,5 \mathrm{~kg}$ & 5 & 15 \\
\hline
\end{tabular}


cases), errant là où il peut trouver de la nourriture, y compris les restes de cuisine, et l'élevage communautaire en petits troupeaux de 20 à 50 têttes menés par un berger, un enfant le plus souvent;

- de par son taux d'urbanisation élevé, et l'absence d'élevage de bovins dans cette région, le Sud Cameroun a des besoins en protéines animales importants, à couvrir dès maintenant. Cette région étant également la zone d'implantation de notre département, nous avons pu la couvrir de plus près par des enquêtes personnelles ou menées sous notre direction par les étudiants que nous formons lors de leurs stages d'études villageoises.

\section{SOCIOLOGIE DE L'ELEVAGE DES OVINS AU SUD-CAMEROUN}

Parler d'élevage est un terme tout à fait impropre pour étudier la manière dont les ovins sont élevés. Bien sûr chaque village traversé laisse entrevoir le spectacle d'un troupeau de moutons de plusieurs dizaines de têtes divaguant au gré de leur fantaisie. Dès le soir tombant on s'aperçoit très vite que cette organisation grégaire est l'œuvre spontanée des animaux qui rentrent chacun passer la nuit sous les auvents de la maison de leur propriétaire. Personne ne s'occupe d'eux et il n'est pas rare que les planteurs " ne connaissent pas " l'effectif de leur cheptel. L'herbe pousse en abondance (1 500 à $4000 \mathrm{~mm}$ de précipitations annuelles) dans ces régions pluvieuses. Les problèmes de nourriture ne se posent pas et tout un chacun y trouve son compte. A vrai dire, cette affirmation était exacte dans le passé car le développement constaté ces dernières années des cultures vivrières ou d'exportation fait maintenant considérer le mouton comme un dévastateur (certes de moins mauvaise réputation que la chèvre) et les palabres entre villageois se multiplient de plus en plus. Les autorités conseillent donc de les attacher, ce qui ne va pas sans poser de problèmes : il faut alors les nourrir et peu de personnes peuvent répondre à la question «que faut-il leur donner?».

De toute façon cet « élevage " n'est pas du tout pratiqué dans un but commercial mais serait plutôt du type «élevage tirelire» que l'on exploite dans certaines occasions exceptionnelles. On peut résumer les quelque 120 enquêtes villageoises effectuées par les chiffres suivants concernant la destination des ovins :
- auto-consommés

35 p. 100 (lors de cérémonies coutumières ou religieuses)

- vendus

20 p. 100

- offerts aux visiteurs

25 p. 100

- dot

20 p. 100

Suivant la taille de la famille, le pourcentage de propriétaires d'ovins s'accroît d'une façon régulière :

TABLEAU $N^{\circ}$ II

\begin{tabular}{|l|c|c|c|c|c|}
\hline $\begin{array}{l}\text { Nombre de personnes dans } \\
\text { la famille }\end{array}$ & 1 & $2-4$ & $5-7$ & $8-15$ & +15 \\
\hline $\begin{array}{l}\text { P.100 de familles élevant } \\
\text { des ovins ou caprins }\end{array}$ & 20 & 40 & 50 & 60 & 70 \\
\hline $\begin{array}{l}\text { Nombre toyen d'animaux } \\
\text { (ovins + caprins) }\end{array}$ & 2,4 & 2,2 & 2,3 & 2,5 & 4.1 \\
\hline
\end{tabular}

Nous avons dû nous résigner à ne pas différencier les ovins et caprins car pour les paysans, il n'y a guère de différence. Dans de nombreux dialectes du Sud, il existe un mot d'usage courant et spontané pour désigner en bloc les deux espèces. Il faut vraiment insister et manier un peu la langue pour arriver à les faire parlet séparément des moutons ou des chèvres. La préférence des éleveurs allait auparavant aux caprins plus rustiques mais beaucoup plus dévastateurs, si bien que le mouvement s'inverse ces dernières années.

Selon nos estimations, les ovins représenteraient actuellement 40 p. 100 du cheptel ovincaprin du Sud Cameroun.

Les résultats d'enquêtes menées dans toute la région Sud font ressortir l'attitude globale des paysans vis-à-vis de l'élevage des ovins-caprins. L'accroissement quasi linéaire de la population "d'éleveurs » avec l'augmentation de la taille de la famille pourrait faire penser à une attitude auto-consommatrice mais le tableau des nombres moyens d'animaux élevés, quasiment constants sauf pour les très grandes familles, détruit cette hypothèse. Il faudrait, selon nous, voir la solution dans l'augmentation des sollicitations extérieures (cadeaux, dots, frais de scolarité...) et avec le nombre croissant des enfants.

La conversation suivante rapportée par l'un de nos étudiants enquêteur est particulièrement symptomatique et généralisable.

«Les animaux que vous voyez ici, au village, 
nous sont d'une très grande importance, ils sont Ia base de toutes nos transactions, leur présence fait notre joie, c'est-à-dire qu'un père de famille est content de voir dans sa cour un grand troupeau.

Vous savez que nous n'avons d'argent ici que pendant la période de vente du café; il peut arriver avant ce temps qu'un membre de la famille tombe gravement malade, alors on vend un certain nombre d'animaux pour avoir l'argent de son traitement. »

\section{Comment envisagez-vous une amélioration?}

« Je ne pense pas qu'il y ait quelqu'un ici au village qui envisage d'apporter des améliorations à notre élevage. Nos grands-parents qui nous ont légué ces animaux faisaient comme nous aujourd'hui et s'en sortaient très bien; je ne vois pas dans quel sens peuvent s'effectuer ces améliorations. »

\section{Il paraît qu'il est très difficile de tuer un animal pour la consommation familiale?}

« Les animaux nous aident à régler la plupart de nos problèmes. Ces animaux nous sont si familiers que l'on préfère acheter de la viande au marché. On les offre comme partie de la dot, ou comme cadeau à des amis à l'occasion de certains événements. »

Les très grandes familles, généralement issues de la polygamie, donc ayant des revenus élevés, peuvent seules pratiquer l'élevage à une certaine échelle; dans ces cas, on peut rencontrer des possesseurs de plus de 10 voire 20 têtes. Pour les autres familles, l'effectif ne varie guère qu'entre 1 et 5 animaux. On peut encore éclairer cet aspect des choses en recherchant qui est propriétaire des ovins. Dans 90 p. 100 des cas, le chef de famille en est propriétaire, la femme et les enfants mâles se partagent les 10 p. 100 restants, les veuves étant considérées comme chef de famille et ayant les « mêmes droits » à posséder ces animaux que les hommes.

La viande de mouton est mangée dans les grandes occasions, généralement sous forme de Ndomba (viande cuite à l'étuvée avec des condiments dans des feuilles de bananiers). Ce plat est très prisé mais demande une longue préparation.

En résumé, malgré de légères variantes suivant les ethnies, l'élevage ovin au Sud Cameroun est presque exclusivement composé d'uni- tés très faibles ( 2 à 4 moutons) menées sans souci de commercialisation et sans connaissance des animaux. L'amélioration de cette spéculation et le passage de l'économie de cueillette à celle de production nécessitera un changement profond des mentalités et une meilleure attention portée aux animaux. Par exemple, il existe bien quelques vocables locaux pour désigner les maladies rencontrées, mais la description des symptômes correspondants est des plus sommaires: perte de poids, animal abattu, peau se couvrant de croûtes...

Quelques grands planteurs de café (moins de 10 à notre connaissance) ont constitué des troupeaux importants dans l'Ouest du pays (100 à 900 têtes). Nous en avons étudié les problèmes parallèlement à notre étude en station. Leurs troupeaux exploitent d'une façon très extensive la strate de couverture des caféières.

Avant d'envisager toute amélioration et de tenter de dégager des perspectives pour l'avenir il convient de faire l'inventaire zootechnique des potentialités de cette race.

\section{VALEUR ZOOTECHNIQUE DE LA RACE DJALLONKE}

\section{Méthodes de travail}

Par des achats successifs dans diverses régions du Sud Cameroun, nous avons constitué un troupeau de mères qui a atteint son effectif maximal vers 1968 ( 80 brebis). Pour des raisons que nous développerons plus loin, nous avons réduit ce troupeau autour de 50 mères.

Afin d'étudier les paramètres zootechniques dans les conditions les plus proches possibles de la pratique normale, nous avons opté pour la lutte continue. Si nous isolons les mères lactantes afin de leur assurer une nutrition adêquate, celles-ci sont toujours avec un bélier.

$\mathrm{Ne}$ disposant d'aucun pâturage réservé à notre élevage ovin, nous avons étudié les potentialités maximales de croissance, d'engraissement et de lactation avec une alimentation de type intensif. Nous sommes bien convaincus que cette méthode est très loin des conditions vulgarisables mais elles nous ont permis d'étudier les maximums à attendre d'élevages intensifs ou extensifs améliorés. Parallèlement, nos enquêtes régulières chez les quelques gros éleveurs nous ont permis d'analyser ces mêmes paramètres en conditions extensives normales. 


\section{Paramètres de reproduction}

\section{Age au premier agnelage}

Globalement sur les 85 données que nous possédons, l'âge moyen au premier agnelage ressort à 16,9 mois, les extrêmes observés étant de 12,2 et 23,7 mois.

Mais on peut corriger ce résultat en constatant qu'en 1969-1970, notre troupeau était trop important pour nos installations et l'âge moyen pour cette période s'élève à 18,4 mois, principalement à cause d'un parasitisme intense et du retard corrélatif de croissance.

Cette correction faite, pour les 6 autres années d'observation, l'âge moyen ressort à 16,3 mois avec un écart type de 2,8 mois.

L'histogramme des fréquences (correction faite) se présente ainsi :

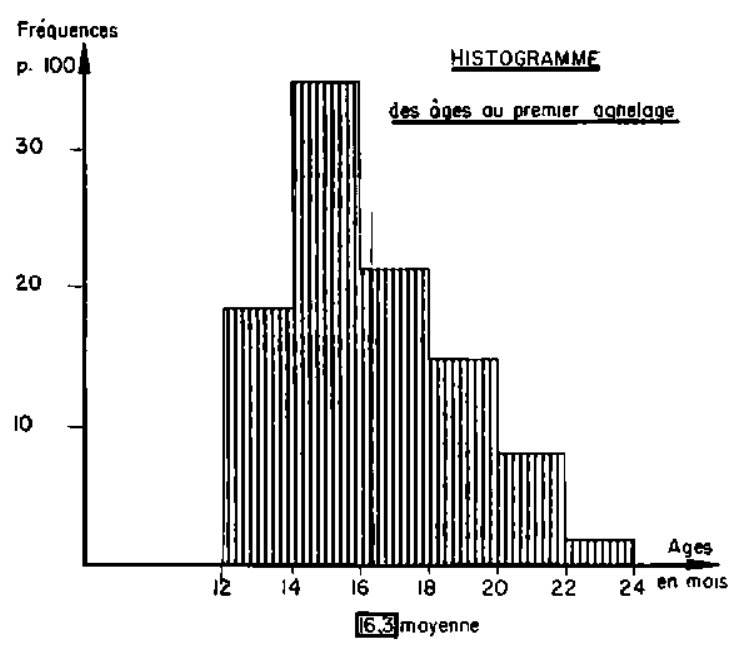

La première saillie fécondante a donc lieu, au plus tôt vers 7 mois, mais en majorité vers 10-12 mois. Comme on pouvait s'y attendre ce paramètre est soumis à l'influence de facteurs nutritionnels et sanitaires, il n'est dès lors pas étonnant que son héritabilité soit faible : 0,19.

\section{Rythme saisonnier de la reproduction}

De 1966 à 1973, nous avons obtenu 561 agnelages répartis en pourcentage sur l'année de la façon suivante :

\begin{tabular}{|l|c|l|r|}
\hline \multicolumn{1}{|c|}{ Mois } & p. 100 & \multicolumn{1}{c|}{ Mois } & p. 100 \\
\hline Janvier & 8 & Juillet & 8,4 \\
Février & 7,3 & Août & 8,2 \\
Mars & 7,3 & Septembre & 7,7 \\
Avril & 8,4 & Octobre & 8,6 \\
Mai & 7,9 & Novembre & 8,8 \\
Juin & 9,1 & Décembre & 10,3 \\
\hline
\end{tabular}

Bien qu'en février et mars 1969, il n'y ait pas eu d'agnelage à la suite d'une tentative de lutte groupée destinée à étudier l'influence du flushing sur les paramètres de reproduction, les agnelages sont statistiquement répartis équitablement sur toute l'année $\left(\mathrm{X}_{11}{ }^{2}=5,19\right.$ - Probabilité 95 p. 100).

Les chaleurs des brebis Djallonké ne sont pas soumises à un rythme saisonnier. Elles peuvent donc reproduire toute l'année. C'est là un de leurs gros avantages, qui est probablement à mettre sur le compte de la proximité de l'équateur et de la faiblesse consécutive des variations du rythme nyctéméral.

\section{Fertilité - Stérilité}

Au cours de notre étude, nous avons mis à la reproduction plus de 140 femelles; une seule s'est avérée complètement stérile : à l'âge de 5 ans elle n'avait toujours rien produit et a été réformée.

Le taux de stérilité totale est donc quasiment négligeable; par contre nous pouvons définir un taux de stérilité temporaire. Comme nous l'étudierons en détail plus bas, l'intervalle entre agnelages successifs tourne en moyenne autour de 8 mois.

Nous considérerons comme temporairement stériles les brebis qui dépassent 13 mois entre deux agnelages. Ce taux de stérilité temporaire est de 3,8 p. 100 pour l'ensemble des 381 intervalles entre mises bas observés.

Un taux de fertilité global de 96 p. 100 pourra donc être pris en compte pour les calculs de productivité et fécondité des brebis Djallonké. Il apparaît normal que ce taux soit élevé puisque cette race n'a fait jusqu'ici l'objet d'aucune sélection. 
Intervalles entre mises bas successives

Rappelons que la lutte ici est continue, une semaine après la mise bas, la brebis lactante est mise en permanence avec un bélier.

L'histogramme des intervalles entre mises bas se présente de la façon suivante :
Les records en ce domaine sont détenus par deux brebis qui ont aligné les intervalles successifs suivants :

a) $8,0-6,6-6,5-6,4-5,1-6,8-6,9-6,3$ : moyenne 6,6 mois;

b) $5,9-7,5-7,2-6,2-6,6-6,0-6,0-7,4$ : moyenne 6,6 mois.

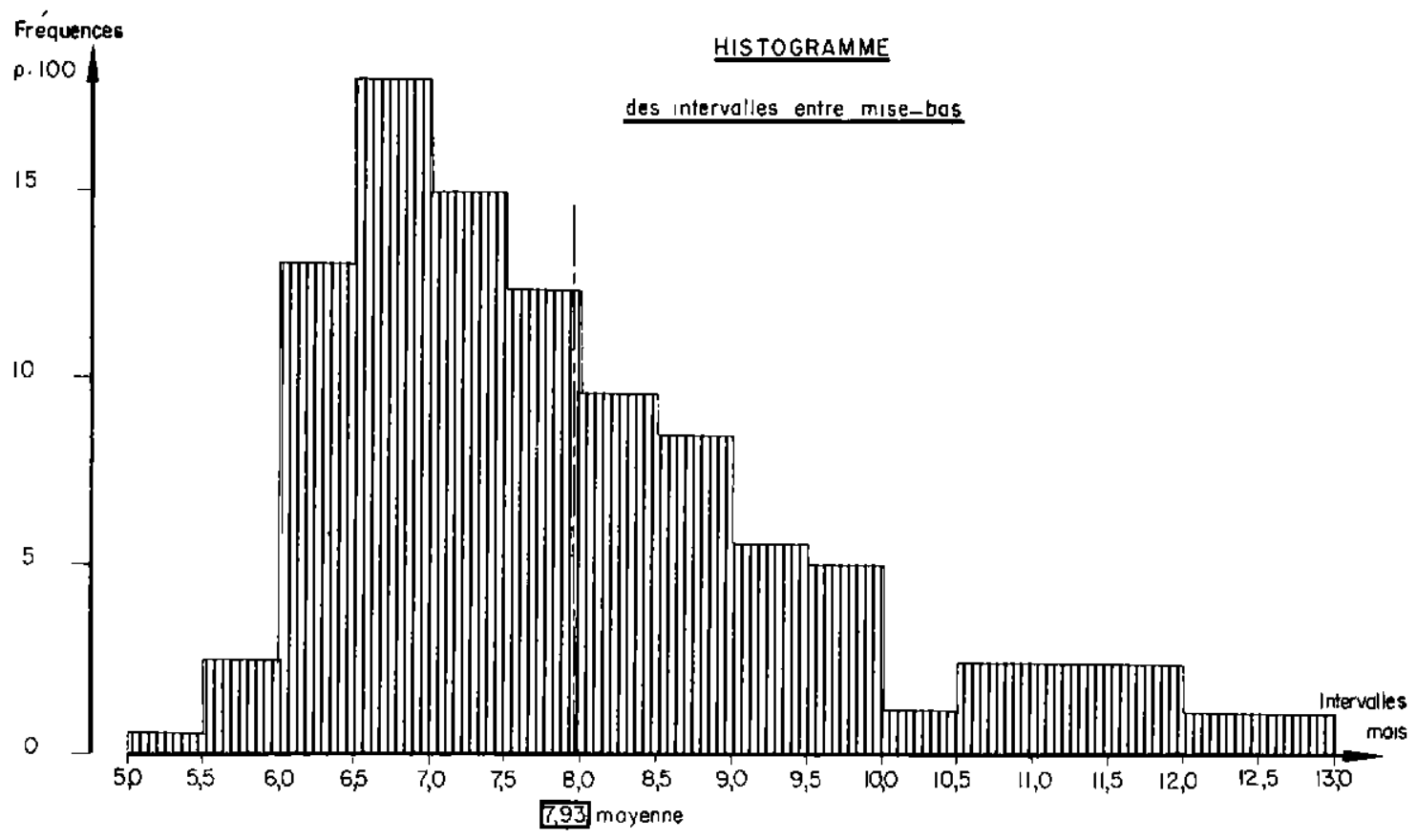

La valeur moyenne pour l'intervalle entre deux mises bas ressort à 7,93 mois (intervalles inférieurs à 13 mois $=96$ p. 100 des cas).

Les brebis Djallonké donnent donc, dans 96 p. 100 des cas, 3 agnelages en 2 ans.

Les brebis sont-elles capables de tenir longtemps ce rythme?

Si nous ne prenons en considération que les données des brebis ayant mis bas au moins 5 fois (maximum observé 11 fois), la valeur de l'intervalle moyen entre mises bas est de 8,12 mois toutes données comprises, c'est-à-dire sans compter à part les stérilités temporaires (intervalles supérieurs à 13 mois).

Sur 60 brebis ayant donné entre 5 et 11 agnelages :

- 14 seulement ont dépassé une fois l'intervalle d'un an et 3 l'ont dépassé 2 fois;

- 28 n'ont jamais dépassé le seuil de 10 mois dont 9 n'ont jamais dépassé 8,5 mois.

\section{Peut-on sélectionner ce caractère?}

L'héritabilité de l'intervalle moyen (calculé sur au moins 3 données), évaluée par la régression fille-mère intra-père, est de 0,46 ; ce qui en fait un caractère très sélectionnable.

Nous avons obtenu une réponse positive à la sélection sur ce caractère puisque cet intervalle moyen, qui était de 8,4 mois chez les brebis d'origine, est tombé à 7,8 mois chez leurs descendants femelles. Pour cela nous avons laissé jouer la sélection naturelle, (les brebis, ayant le cycle le plus court, donnent plus de descendants et distribuent donc plus leurs gènes dans le troupeau) en permettant aux génotypes de s'exprimer complètement par une alimentation plus adaptée aux diverses périodes du cycle de reproduction.

Cette héritabilité semble confirmée par le fait que deux sœurs jumelles monozygotes ont donné chacune 8 agnelages jamais séparés par plus de 2 semaines l'un de l'autre. 


\section{Prolificité}

Ce critère défini comme le nombre dagneaux nés pour cent mises bas est globalement de 117 pour cette race, avec des variations annuelles extrêmes allant de 107 à 120 (8 années d'observations).

En fait, ce taux est de 100 pour les primipares, 103 en deuxième agnelage et de 120 pour les agnelages suivants.

Nous n'avons jamais observé de naissance de triplés ni en station ni dans les élevages particuliers. L'étude des variations individuelles des 60 brebis ayant agnelé au moins 5 fois donne la répartition suivante :

\begin{tabular}{|c|r|}
\hline Prolificité & Pourcentage \\
\hline $100-110$ & 54,0 des brebis \\
$110-120$ & 14,6 des brebis \\
$120-130$ & 12,5 des brebis \\
$130-140$ & 6,3 des brebis \\
$140-150$ & 6,3 des brebis \\
$150-160$ & 4,2 des brebis \\
$160-170$ & 2,1 des brebis \\
\hline
\end{tabular}

Environ la moitié des brebis n'a jamais donné de jumeaux dans leur carrière reproductrice.

Les plus prolifiques sont les deux sœurs monozygotes qui ont donné l'une 5 fois des jumeaux, l'autre 4 fois sur 8 agnelages. Ellesmêmes issues d'une lignée prolifique, leur descendance s'avéra aussi très prolifique. L'héritabilité de la prolificité mesurée sur au moins 4 agnelages est de 0,26 , ce qui confirme des travaux antérieurs menés sur des races françaises par l'un des auteurs (non publiés).

On pourrait s'étonner qu'une race aussi rustique soit si peu prolifique; cependant, dans les conditions naturelles, ces animaux sont difficilement capables d'allaiter plus d'un agneau. Même bien nourris ils conservent cette faible potentialité acquise par équilibre naturel et l'essai de flushing que nous avons mené sur 75 brebis n'a pas répondu positivement : la prolificité est restée identique.

\section{Corrélation, prolificité -}

Intervalle entre mises bas

On peut s'attendre à une corrélation phénotypique négative: les plus prolifiques étant celles à cycle le plus long pour permettre de récupérer des réserves.

En fait, cette corrélation n'est que de : 0,17 , ce qui est très faible. Il n'y a donc pas incompatibilité à sélectionner ces deux caractères pour obtenir une fécondité (nombre d'agneaux nés par mère et par an) supérieure. Nous ne disposons malheureusement par suffisamment de données pour calculer la corrélation génotypique qui nous permettrait d'être parfaitement renseigné sur le choix des critères à améliorer.

A notre avis, il ne semble pas y avoir incompatibilité pour l'amélioration conjointe de ces deux caractères.

L'attitude des 5 brebis les plus prolifiques et des 5 brebis à intervalle moyen le plus court donne le tableau suivant :

\begin{tabular}{|c|c|}
\hline Intervalle & Prolificité \\
\hline & $\begin{array}{c}\text { les } \begin{array}{c}5 \text { brebis à cycle } \\
\text { le plus court }\end{array} \\
6,48 \text { mois }\end{array}$ \\
6,57 & 142 \\
6,68 & 100 \\
7,08 & 128 \\
7,15 & 143 \\
\cline { 2 - 3 } & 127 p. 100 \\
6,79 mois & les 5 brebis les plus \\
Moyennelifiques \\
& 167 \\
7,46 & 157 \\
7,62 & 150 \\
8,15 & 150 \\
8,17 & 143 \\
7,15 & 153 p. 100 \\
\hline 7,71 mois & \\
\hline Moyenne & \\
\hline
\end{tabular}

\section{Fécondité}

Définie comme le nombre d'agneaux nés par an et par brebis adulte, la fécondité moyenne des brebis Djallonké adultes peut se calculer ainsi :

$$
\begin{gathered}
1 \times 0,96 \times \frac{12 \text { mois }}{8 \text { mois }} \times 117 \\
\text { (fertilité) } \quad \begin{array}{c}
\text { (intervalle } \\
\text { entre mise-bas) }
\end{array} \\
=1,68 \text { agneaux nés } / \text { an } / \text { mère } \\
\text { (prolificité) }
\end{gathered}
$$

Ce qui en fait une race assez féconde malgré sa faible prolificité. 
Les 10 brebis les plus fécondes ont atteint les niveaux de :

$2,68-2,64-2,47-2,40-2,39-2,21-2,20-$ $2,19-2,15-2,12$.

(La première et la troisième sont les deux jumelles monozygotes.)

\section{Longévité}

L'évaluation de ce paramètre se heurte à de nombreux problèmes qui ont perturbé le cours normal de la vie des brebis.

- L'introduction d'un nouveau noyau important de femelles en 1968 a provoqué une mortalité accrue, vraisemblablement due, comme nous le discuterons dans le chapitre sanitaire, à l'apport de facteurs pathogènes étrangers.

- En 1970, à la suite d'un congé de maladie d'un chercheur responsable, plus de 25 p. 100 des brebis se sont "évaporées» dans la nature.

- Enfin, la durée de vie ne peut être évaluée que pour des animaux nés à la station car personne ne tient de cahier de bergerie dans les autres élevages. Nous ne disposons pas de données en nombre suffisant pour évaluer correctement la longévité.

Disons pour fixer les idées que 9 brebis ont dépassé les 10 agnelages et que la moyenne des brebis achetées adultes (âges et nombres d'agnelages antérieurs inconnus) est de 6,2 agnelages.

Pour nos calculs ultérieurs, nous tablerons donc sur une moyenne de 7 agnelages par brebis soit un âge de réforme de 6 à 6,5 ans.

Le taux de mortalité moyen pour les brebis adultes (plus de 2 ans) est d'environ 4 p. 100 par an pour l'ensemble du troupeau adulte. Il ne faut pas oublier que nous ne réformons pas les femelles et que, pour des raisons expérimentales, nous les gardons jusqu'à leur mort naturelle causée dans 90 p. 100 des cas par des toxémies ou des abattages consécutifs à une impossibilité de reproduire (renversement de vagin par exemple); le reste étant des accidents dus aux manipulations nombreuses dont elles sont l'objet.

\section{Paramètres d'élevage et d'engraissement}

\section{Poids à la naissance}

Nous ne disposons pas de chiffres pour les poids à la naissance des ovins élevés en extensif mais, d'après nos estimations, nous avons tout lieu de croire que ce poids est un peu inférieur aux données récoltées dans notre station, résumées dans le tableau III en comparaison avec celles de Sotuba.

Les extrêmes varient, pour les mâles nés simples de 1,7 à $4,5 \mathrm{~kg}$; dans ces chiffres, nous n'avons pas tenu compte séparément des agneaux de primipares toujours plus petits en moyenne. Avec une bonne supplémentation avant la mise-bas, nous avons pu accroître le poids moyen des mâles à 2,7 ; chiffre que l'on devrait pouvoir obtenir facilement en menant bien l'alimentation des brebis mais qui, pour des raisons économiques, est impossible à réaliser actuellement.

\section{Valeur laitière des mères}

Nous avons choisi comme indice de la valeur laitière des mères, le gain moyen journalier de leur(s) agneau(x) entre 0 et 30 jours, nous inspirant ainsi des normes de calcul de la Fédération Nationale ovine de France qui préconise l'utilisation du gain 10 - 30 jours. Entre ces deux paramètres, la corrélation est de 0,82 et notre choix a l'avantage de simplifier les calculs.

Pour les mêmes raisons que ci-dessus il est difficile d'avoir une idée très précise de la valeur laitière des brebis menées en extensif. Cet indice est de l'ordre de 80 grammes par

TABLEAL $\mathrm{N}^{\circ}$ III

\begin{tabular}{|c|c|c|c|c|c|c|}
\hline Station & \multicolumn{4}{|c|}{ Nkolbisson $(1965-73)$} & \multicolumn{2}{|c|}{ Sotuba $(1953-57)$} \\
\hline Sexe & \multicolumn{2}{|c|}{$M$ a $1 \mathrm{e}$} & \multicolumn{2}{|c|}{$\mathrm{F}$ e me $1 \mathrm{e}$} & Mâle & Feme11e \\
\hline $\begin{array}{l}\text { Nombre de } \\
\text { naissances }\end{array}$ & Simple & Doub1e & Simple & Double & \multicolumn{2}{|c|}{ non précisẻ } \\
\hline $\begin{array}{l}\text { Poids à } 1 \mathrm{a} \\
\text { naissance }(\mathrm{kg})\end{array}$ & 2,5 & 2,0 & 2,3 & 1,8 & 2,1 & 1,7 \\
\hline
\end{tabular}


jour. En station, nous avons obtenu les résultats suivants :

TABLEAU $\mathbb{N}^{\circ} \mathrm{IV}$

\begin{tabular}{|c|c|c|c|c|}
\hline Période & \multicolumn{2}{|c|}{$65-69$} & \multicolumn{2}{|c|}{$70-73$} \\
\hline Nombre d'agneaux & 1 & 2 & 1 & 2 \\
\hline $\begin{array}{l}\text { G.M.J. 0-30 jours } \\
\text { (en grammes) }\end{array}$ & 115 & 85 & 134 & 112 \\
\hline
\end{tabular}

Pendant la première période, les brebis lactantes étaient peu supplémentées avec un concentré valable pour tous les ovins $(90 \mathrm{~g}$ MAD/UF). Par contre durant la seconde période (1970-1973) les brebis lactantes ont reçu un concentré plus riche en protéines (135 g MAD/UF) donc plus conforme à leurs besoins spécifiques. En plus, nous avons commencé à sélectionner les agnelles sur la valeur laitière de leur mère début 1969.

Bien alimentées, plusieurs mères sont capables d'assurer à leur agneau un gain moyen de 200 à $250 \mathrm{~g} /$ jour. Les primipares sont normalement moins laitières et un gain moyen de $95 \mathrm{~g} / \mathrm{j}$ peut être retenu pour elles. La durée de lactation pour ces brebis a été évaluée par le Centre de Recherches Zootechniques de Sotuba à 117 jours \pm 6 et la production moyenne de lait à $87 \mathrm{~kg}$ chez les mères supplémentées.

\section{Croissance des agneaux}

La Fédération Nationale ovine utilise le gain moyen journalier 30-90 jours pour évaluer la croissance des agneaux. Vu le peu de précocité de la race Djallonké, nous avons adopté pour ce paramètre le gain moyen journalier $30-150$ jours qui tient mieux compte des conditions réelles de croissance de ces animaux.
Les résultats que nous avons obtenus tant en station qu'en conditions d'élevage extensif contrôlé sont résumés dans le tableau $\mathrm{V}$.

Dans notre station de Nkolbisson, nous avons utilisé successivement les suppléments suivants :

1. Tous les animaux étaient menés ensemble (sevrage non dirigé) et ils étaient tous supplémentés avec le même concentré : (maïs $60 \mathrm{p}$. 100 , tourteau de coton 20 p. 100 , raffles de maïs 20 p. 100) .

2. Agneaux menés avec leur mère jusqu'à 4-5 mois puis mis seuls en parc d'engraissement - concentré : 50 p. 100 drèche desséchée 50 p. 100 mélasse distribué dans un dispositif de creep-feeding avant le sevrage.

3. Même technique mais concentré plus protéique avec 2 p. 100 d'urée en plus des drèches et mélasses, également distribué dans un dispositif de creep-feeding avant sevrage.

Ces résultats sont en fait la résultante de trois facteurs dont l'influence spécifique est parfois difficile à évaluer :

- la sélection sur le format et sur la valeur laitières des mères;

- la maîtrise du parasitisme cas (2) et (3);

- la nature du concentré - entre (2) et (3) les deux premiers facteurs ont peu influé; ces deux essais se sont succédé à 6 mois d'intervalle. Dans ces conditions, on peut estimer entre 10-12 UF par $\mathrm{kg}$ de gain (entretien compris) les besoins de ces animaux en croissance.

Cette différence de croissance se traduit évidemment sur l'âge moyen de la commercialisation (au poids de $20 \mathrm{~kg}$ minimum) (Tabl. VI).

TABLEAU N* $\mathrm{V}$

\begin{tabular}{|l|c|c|c|c|c|c|}
\hline \multicolumn{2}{|c|}{ ZON E } & $\begin{array}{c}\text { M a l i } \\
\text { (Sotuba) }\end{array}$ & $\begin{array}{c}\text { Sud-Cameroun } \\
\text { extensif }\end{array}$ & \multicolumn{3}{c|}{ Nkolbisson } \\
\hline \multicolumn{2}{|c|}{ Supplémentation } & Probablement ? & sans & (1) & $(2)$ & $(3)$ \\
\hline \begin{tabular}{l} 
Gain $\begin{array}{l}\text { moyen/j } \\
30-150 \mathrm{j}\end{array}$ \\
\cline { 2 - 7 }
\end{tabular} & Males & $59 \mathrm{~g}$ & $52 \mathrm{~g}$ & $64 \mathrm{~g}$ & $72 \mathrm{~g}$ & $85 \mathrm{~g}$ \\
\hline
\end{tabular}

TABLEAU $N^{\bullet} \mathrm{VI}$

\begin{tabular}{|c|c|c|c|c|c|}
\hline \multirow{2}{*}{\multicolumn{2}{|c|}{ Conditions }} & \multirow{2}{*}{ Extensif } & \multicolumn{3}{|c|}{ Station Nkolbisson } \\
\hline & & & (1) & (2) & (3) \\
\hline $\begin{array}{l}\text { Age } \\
\text { P. } 20 \mathrm{~kg} \\
\text { (mois) }\end{array}$ & $\left\{\begin{array}{l}\text { Males } \\
\text { Femelles }\end{array}\right.$ & $\begin{array}{l}12-14 \text { mois } \\
14-16 \text { mois }\end{array}$ & $\begin{array}{r}9,6 \mathrm{~m} \\
10,9 \mathrm{~m}\end{array}$ & $\begin{array}{r}8,7 \mathrm{~m} \\
10,2 \mathrm{~m}\end{array}$ & $\begin{array}{l}7,3 \mathrm{~m} \\
8,5 \mathrm{~m}\end{array}$ \\
\hline
\end{tabular}


A l'heure actuelle 33 p. 100 des agneaux atteignent $20 \mathrm{~kg}$ avant l'âge de 6 mois.

\section{Paramètres de sevrage}

D'après les études menées à Sotuba, les brebis donnent du lait pendant environ 4 mois mais cette production ne semble pas provoquer d'anœstrus de lactation prolongé.

La précocité du sevrage n'a aucun effet sur le rythme des mises bas; nous avons établi ce fait en pratiquant pendant deux années le sevrage précoce $(2-2,5$ mois) pour essayer de diminuer l'intervalle entre mises bas; en fait celui-ci n'a pas varié quel que soit l'âge au sevrage.

En brousse, les agneaux se sèvrent naturellement vers 5 mois, à un poids moyen de $11-12 \mathrm{~kg}$.

Finalement, après maints essais, nous avons retenu aussi l'âge de 4,5 à 5 mois pour lequél nous n'observons pas d'arrêt de croissance après sevrage. En supplémentant les mères lactantes et les agneaux, nous atteignons le poids au sevrage de $15 \mathrm{~kg}$ pour les mâles et $14 \mathrm{~kg}$ pour les femelles et 16 p. 100 des mâles atteignent le poids de commercialisation $(20 \mathrm{~kg})$ avant sevrage. Seule cette spéculation: commercialisation d'agneaux gris, pourrait rentabiliser les concentrés pour peu que les carcasses soient payées à la qualité par les bouchers, ce qui n'est pas le cas actuellement et ce qui, de toute façon, resterait une solution très particulière et non vulgarisable à grande échelle.

Les problèmes d'alimentation autour du sevrage sont loin d'être résolus et nous les aborderons plus loin.

\section{Mortalité}

A la naissance, chaque brebis produit donc annuellement 1,68 agneaux/an desquels il faut déduire les mortalités avant un an pour obtenir la productivité annuelle nette par brebis.

\section{Mortalité avant un an}

Comme ci-dessus, nous diviserons les huit années d'observation en deux périodes : 19661970 correspond à la période de création du troupeau, d'introduction d'animaux étrangers et de mise au point de la technicité d'élevage, 1971-1974 pouvant être considéré comme la période d'exploitation normale:
TABLEAU $N^{\circ}$ VII

\begin{tabular}{|c|c|c|}
\hline \multirow{2}{*}{ C a u s e $s$} & \multicolumn{2}{|c|}{ Pourcentage de mortalité } \\
\hline & $1966-70$ & $1971-74$ \\
\hline $\begin{array}{l}\text { Mort-nës-Malformés } \\
\text { Faibles }\end{array}$ & 3,3 p. 100 & $4,3 \mathrm{p} \cdot 100$ \\
\hline $\begin{array}{l}\text { Agalaxie maternelle } \\
\text { Toxicoses }\end{array}$ & $\begin{array}{ll}5,0 & 11 \\
3,3 & 11\end{array}$ & $\begin{array}{l}3,8 \\
4,5\end{array}$ \\
\hline $\begin{array}{l}\text { Parasitisme } \\
\text { Maladies respiratoires }\end{array}$ & $\begin{array}{l}6,1 \\
5,8\end{array}$ & $\begin{array}{r}1,0 \\
10,1\end{array}$ \\
\hline Métëorisations & 1,0 & " \\
\hline $\begin{array}{l}\text { Non determinee avec } \\
\text { certitude }\end{array}$ & 7,7 & 0,4 \\
\hline $\begin{array}{l}\text { Mortalite avant } 1 \text { an } \\
\text { (tous modes de nais- } \\
\text { sance) }\end{array}$ & 32,2 & 24,1 \\
\hline Mortalitê des jumeaux & $\begin{array}{l}44 \\
29\end{array}$ & $\begin{array}{l}33 \\
21\end{array}$ \\
\hline
\end{tabular}

La mortalité globale avant un an a donc diminué de 8 points entre ces deux périodes; à cela plusieurs explications :

- Le troupeau n'a pas reçu d'animaux étrangers durant la dernière période, donc le microbisme de bergerie ne s'est pas modifié et les jeunes vivaient par conséquent dans une meilleure ambiance sanitaire, à laquelle ils étaient adaptés.

- Le parasitisme a été efficacement maîtrisé malgré l'emploi de parcours très réduits $(0,5$ hectare pour les quelque 60 sevrés et mères suitées). Faute de place, il n'était pas question de faire une rotation des parcours.

- Le nouveau fléau à combattre est représenté par les maladies respiratoires malgré l'utilisation répétée de vaccins polyvalents dans la vie d'un agneau (à 4,6 et 20 semaines).

Ce chiffre de mortalité global de 24 p. 100 est encore trop élevé par rapport à ceux observés dans des élevages de races rustiques européens. Malgré les soins et vaccinations dont notre troupeau fait l'objet en station, nous obtenons une mortalité de même ordre de grandeur qu'en brousse. D'après nos sondages, on peut l'estimer à 30 p. 100 pour les jeunes mais il s'est avéré impossible d'en déterminer les causes : les planteurs employant des expressions très vagues pour décrire les symptômes. Il est plus que probable que notre mortalité sera notablement diminuée le jour où nous disposerons d'installations et de superficies adéquates pour diminuer les densités des parcs. 


\section{Mortalités après un an}

Les agnelles d'élevage (de 1 à 2 ans) meurent dans la proportion de $5-8$ p. 100 , la quasitotalité de maladies respiratoires.

Quant aux brebis adultes, leur taux de mortalité annuel est de l'ordre de 4 p. 100; la plupart étant dues à des toxicoses ou accidents de la reproduction, ce qui ne saurait nous étonner vu la rapidité du cycle de reproduction que suivent certaines brebis. Ces accidents ne surviennent jamais avant le $4^{\mathrm{e}}$ agnelage mais, rappelons-le, certaines brebis ont mis bas plus de 10 fois au rythme normal sans accident.

\section{Valeur des carcasses}

Nous ne disposons que de relativement peu de données (23) pour faire une évaluation exacte des rendements des agneaux abattus. Nos clients, Camerounais en majorité, préfèrent pour de multiples raisons : religieuses ou traditionnelles, emporter leur animal sur pied. Pour un mâle d'environ $20 \mathrm{~kg}$ de poids vif (à jeun de 18 heures) nous pouvons donner les résultats moyens d'abattage suivants:

Poids vif moyen . . . . $20,200 \mathrm{~kg}$

Tête $1,630 \mathrm{~kg}$ $(1,300$ sans cornes) (1,800 avec cornes)

Pattes . . . . . . . $0,560 \mathrm{~kg}$

Peall . . . . . . . 1,470 kg

Viscères non vidés . . . . $5,100 \mathrm{~kg}$

Testicules . . . . . . . $0,190 \mathrm{~kg}$

Foie, rein, cceur, poumons . $0,950 \mathrm{~kg}$

Carcasse non ressuyée . . . $8,700 \mathrm{~kg}$

Pertes (sang, urines...) . . 1,600 kg

soit un rendement carcasse poids vif $=43,1$ p. 100 avant ressuyage

$$
\frac{\text { carcasse }+ \text { abats }}{\text { poids vif }}=47,8 \text { p. } 100
$$

Une distribution suivant le poids vif à l'abattage donne les rendements chauds suivants :

TABLEAU $\mathrm{N}^{\circ}$ VIII

\begin{tabular}{|c|c|c|}
\hline Poids vif & Sans abats & Avec abats \\
\hline $18 \mathrm{~kg}$ & $40 \mathrm{p} .100$ & $44,5 \mathrm{p} .100$ \\
\hline $20 \mathrm{~kg}$ & 43 & 480 \\
\hline $22 \mathrm{~kg}$ & 47 & 51,5 \\
\hline $28 \mathrm{~kg}$ & $48,5 \quad "$ & 53,5 \\
\hline
\end{tabular}

\section{L'UNITE ZOOTECHNIQUE DE PRODUCTION ET SON AMELIORATION}

\section{Structure actuelle de l'unité zootechnique}

En station

La base de calcul est une brebis adulte, c'est-à-dire une femelle à partir de son deuxième agnelage, donc âgée de 2 ans, jusqu'à sa mort ou sa réforme.

Les calculs de la productivité de cette unité zootechnique utiliseront les paramètres que nous avons évalués dans le chapitre précédent à savoir :

taux de fertilité . . . . . 0,96

taux de prolificité : primipare 1,00

adulte . 1,16

intervalle entre mise bas . . 8,0 mois

âge au premier agnelage . . 16,0 mois (pour simpl.)

taux de renouvellement annuel 25 p. 100

taux de mortalité avant un an 24 p. 100

taux de mortalité de un à 2 ans 8 p. 100

âge à la commercialisation . 8,0 mois

Tous les 8 mois une brebis adulte donne naissance à $1 \times 0,96 \times 1,16=1,11$ agneaux dont 76 p. 100 survivront jusqu'à la commercialisation, soit : $1,11 \times 0,76=0,84$ ou 0,42 mâles,

0,42 femelles.

Nous devrons garder chaque année des agnelles pour renouveler les mères réformées soit par chaque 8 mois et en tenant compte de la mortalité :

$$
0,25 \times \frac{8}{12} \times 1,08=0,18 \text { agnelles }
$$

Par période de 8 mois, nous pouvons donc commercialiser 0,42 mâles $+(0,42-0,18)$ femelles $=0,66$ agneaux dont 30 p. 100 , soit 0,20 n'atteindront les $20 \mathrm{~kg}$ requis qu'entre 8 et 12 mois.

A un moment donné, compte tenu des agnelages se répartissant sur toute l'année, notre troupeau aura ainsi la structure suivante:

1 brebis adulte

0,03 béliers

0,18 agnelles 


\section{0,17 primipares}

0,84 agneaux de moins de 8 mois

0,20 agneaux de plus de 8 mois

0,09 agneaux de moins de 8 mois nés des primipares

soit : 2,51 animaux par brebis adulte présente, structure que nous désignerons dans la suite comme l'unité zootechnique de station ou UZ station.

Ce chiffre, bien que pouvant apparaître théorique, n'en donne pas moins une estimation très proche de la réalité : ainsi au jour où nous écrivons cet article, nous avons 41 brebis adultes; notre troupeau devrait donc comprendre $41 \times 2,51=103$ animaux; or l'effectif actuel est de 106 dont il faut déduire 2 jeunes mâles commercialisables de plus de $20 \mathrm{~kg}$ dont la vente ramènerait l'effectif normal à 104 .

A de nombreuses reprises depuis 2 ans, nous avons vérifié la validité de ces calculs.

En pratique, les planteurs ne savent pas différencier les primipares des adultes; il convient donc de ramener notre unité zootechnique au nombre de femelles ayant agnelé. Dans ces conditions, nous obtiendrons une unité améliorée : $\mathrm{UZ}$ améliorée $=2,24$ animaux par brebis présente.

\section{En conditions extensives}

En extensif traditionnel, ces chiffres doivent être corrigés car les agneaux n'atteignent le poids de «commercialisation » que bien après 8 mois.

On peut raisonnablement estimer que les paramètres de reproduction sont sensiblement les mêmes qu'en élevage amélioré.

D'après nos observations, nous devrons modifier principalement les paramètres suivants :

— taux de mortalité avant 8 mois : 35 p. 100

— taux de mortalité de 8 à 16 mois : 10 p. 100

- pourcentage de commercialisation avant 8 mois :

0 p. 100

La structure de l'unité zootechnique extensive (UZ ext.) serait donc voisine de :

1 brebis .

40 p. 100

0,10 bélier (beaucoup d'éleveurs conservent 1 bélier. .

4 p. 100

0,72 agneaux de moins de 8 mois
0,65 agneaux de plus de 8 mois . 26 p. 100

soit : UZ ext. $=2,47$ animaux par brebis présente.

Nos enquêtes en milieu villageois ont fait ressortir pour des «troupeaux de village » des chiffres de cet ordre: de 2,35 à 2,51.

\section{Productivité de ces unités zootechniques}

En milieu villageois

Parmi les 0,65 agneaux de plus de 8 mois, 0,18 agnelles devront être conservées pour maintenir le troupeau. Il restera donc chaque 8 mois, 0,47 animaux à «commercialiser » soit :

0,70 agneaux par an et par UZ extensive

ce qui représente un taux annuel de commercialisation de 28 p. 100.

Nos enquêtes villageoises vérifient ces chiffres puisque nous avons relevé :

- des productivités de 0,64 à 0,75 agneaux/ an/brebis;

- des taux de commercialisation de 24 à 31 p. 100.

En brousse, un agneau d'environ $20 \mathrm{~kg}$ est vendu 3000 F CFA (au Sud-Cameroun) et une brebis autour de $4000 \mathrm{~F} \mathrm{CFA}$.

Le revenu brut annuel de l'UZ extensive est donc de :

$$
\begin{gathered}
0,70 \underset{(\text { agneau })}{\times 3000}+\underset{(\text { brebis réforme })}{0,10 \times 4000} . \\
=2500 \mathrm{~F} / \text { an } / \text { brebis }
\end{gathered}
$$

\section{En station}

L'UZ améliorée permet de commercialiser chaque année:

\section{0,99 agneaux par an et par UZ am.}

correspondant à un taux annuel de commercialisation de 44 p. 100. En élevage amélioré, le revenu monétaire brut est de: $3350 \mathrm{~F} / \mathrm{an} /$ brebis, soit un accroissement de 34 p. $100 \mathrm{du}$ revenu brut par brebis. Ce même revenu s'établit à 3800 F CFA/an/brebis adulte pour l'UZ de station; chiffre que nous vérifions également par la pratique puisque nos recettes annuelles sont de l'ordre de $160000 \mathrm{~F}$ CFA pour un effectif de brebis adultes oscillant entre 40 et 45 têtes. 
Besoins nutritionnels de ces unités zootechniques

\section{Paramètres utilisés}

Brebis en lactation

En période de lactation, la production et les besoins journaliers maximaux s'élèvent à :

TABLEAU $N^{\circ} I X$

\begin{tabular}{|c|c|c|}
\hline Besoins & Exte d'élevage & Amélioré \\
\hline $\begin{array}{c}\text { G.M.j. (en g) } \\
(0-30 j .)\end{array}$ & 80 & 150 \\
\hline kg lait/j & 0,7 & 1 \\
\hline U.F./j & 0,85 & 1,10 \\
\hline G. M.P.D./U.F. & $100-105$ & $110-115$ \\
\hline
\end{tabular}

D'après nos évaluations, le niveau de consommation alimentaire de ces ovins est de :

\section{3,7 à $4,2 \mathrm{~kg}$ de matière sèche pour $100 \mathrm{~kg}$ de poids vif.}

Un animal de $30 \mathrm{~kg}$ pourra donc ingérer de 1,10 à $1,25 \mathrm{~kg}$ de matière sèche par jour. Avec une alimentation exclusivement basée sur les fourrages, les brebis menées en extensif arrivent tout juste à couvrir leurs besoins en période de pointe de lait avec une herbe d'exceIlente qualité, soit au moins à $0,68 \mathrm{UF} / \mathrm{kg}$ de matière sèche, chiffre qui est rarement atteint par les fourrages locaux hormis les légumineuses et quelques excellentes graminées tropicales.

\section{Agneaux en croissance}

Le même genre de calcul conduit à démontrer qu'avec une alimentation exclusivement fourragère les agneaux ne peuvent après 1 mois dépasser un gain moyen journalier de 40 - 50 grammes, mểme avec des fourrages d'excellente qualité. En effet, d'après nos évaluations, les besoins de croît s'élèvent autour de : 0,5 à 0,6 UF par 100 grammes de gain de poids vif contre 0,25 à 0,3 dans les tables européennes.

\section{Charges à thectare}

Comme nous venons de le démontrer, les performances atteintes en extensif sont presque les limites de production possible de ces ovins en pâture exclusive. Pour estimer les charges applicables en élevage amélioré, il nous faudra obligatoirement concevoir une nourriture d'appoint que nous déterminerons plus loin.

\section{Charges en extensif}

Chaque Unité Zootechnique extensive a un besoin annuel d'environ 460 UF.

Si nous estimons entre 3000 et 4000 UF la production annuelle des pâturages tropicaux guinéens du Sud-Cameroun non améliorés mais bien menés, ceux-ci pourront supporter :

entre 6,5 et 8,5 mères suitées à l'hectare.

Charges en amélioré

Le pâturage ne peut pas couvrir seul les besoins des animaux, particulièrement pendant les 2 premiers mois de lactation durant lesquels les besoins journaliers dépassent 0,9 UF, ce qui supposerait avec un fourrage d'excellente qualité un niveau d'ingestion supérieur à $4,3 \mathrm{~kg}$ de matière 'sèche par $100 \mathrm{~kg}$ de poids vif.

De même, ce pâturage est incapable d'assurer la croissance des agneaux au rythme de $70-80 \mathrm{~g}$ de gain moyen journalier.

Avec ces performances, le fourrage ne peut être utilisé que pour couvrir 305 des 430 UF nécessaires à l'unité zootechnique améliorée chaque année.

Une charge de 10 à 13 brebis suitées à l'hectare pourra donc être utilisée.

Dans notre station, un pâturage de 2,5 hectares implanté en Brachiaria ruziziensis suffit largement à couvrir les besoins fourragers de nos 49 unités améliorées, soit une charge effective de près de 20 mères suitées à l'hectare. Ce pâturage est bien sûr fumé régulièrement et produit de l'ordre de 6500 UF par hectare et par an 'en zéro grazing.

\section{Coûts de productions de ces unités zootechniques}

\section{Unité zootechnique extensive}

Traditionnellement, les ovins sont laissés en liberté dans la brousse, où ils exploitent les jachères. Dans les régions de forte densité de population donc de forte culture, les moutons sont attachés et plus exceptionnellement parqués pendant la saison des plantations (avril à nọvembre). Même dans ce dernier cas, les propriétaires se contentent soit de déplacer leurs moutons chaque matin, soit de leur couper du fourrage qu'ils jettent dans les enclos. 
On leur donne aussi des restes de cuisine et d'épluchage mais ceux-ci sont le plus souvent réservés en priorité aux porcs. La ration s'améliore un peu en période de récolte avec la distribution des résidus de grains et tubercules non consommables dans l'alimentation humaine.

Les soins sont quasiment inexistants. Dans ces conditions, les coûts de production en élevage extensif traditionnel sont pratiquement nuls.

Dans le Sud du Cameroun, la taille moyenne des exploitations est de l'ordre de 2 hectares par famille, les jachères occupent un maximum de 0,4 hectare par exploitation, chaque famille pourra donc entretenir sur ses terres de l'ordre de 2,5 brebis suitées et en retirer un surplus de revenu de quelque $6200 \mathrm{~F}$ CFA par an; ce qui n'est pas négligeable lorsque l'on sait que le revenu annuel moyen des planteurs est de l'ordre de 40000 F CFA même avec des cultures industrielles (résultat de 120 enquêtes sur 7 exploitations chacune).

\section{Unité Zootechnique améliorée}

\section{Habitat}

Il est certain que l'êlevage amélioré tel que nous l'avons testé en station suppose un minimum d'infrastructure pour que chaque type d'animal puisse être mené selon ses besoins propres. La construction d'une bergerie compartimentée s'impose donc. Même construite en matériaux locaux, il n'est pas envisageable de descendre en dessous de $700 \mathrm{~F}$ le mètre carré couvert et installé pour avoir un minimum de commodité et d'hygiène soit, à raison de 2 mètres carrés par UZ, un investissement de $1400 \mathrm{~F}$ par UZ amortissables sur 5 ans, d'où une charge annuelle de $280 \mathrm{~F}$ par UZ et par an.

\section{Frais vétérinaires}

Un programme de vaccination et de prévention des parasitoses doit être mis en cuvre pour diminuer la mortalité et accroître l'efficacité alimentaire. Nous étudierons plus bas les solutions que nous préconisons après 8 ans de recherches. Un minimum de $150 \mathrm{~F}$ par an et par UZ devra être prévu.

\section{Supplémentation alimentaire}

Les fourrages apportent $310 \mathrm{UF}$ à notre UZ améliorée, le lait environ 20 UF aux agneaux après 6 semaines, les suppléments alimentaires devront donc apporter autour de $100 \mathrm{UF}$.
Le supplément de productivité de l'UZ améliorée par rapport aux conditions extensives a été évalué à $850 \mathrm{~F} \mathrm{CFA} \mathrm{par} \mathrm{an} \mathrm{desquels} \mathrm{il} \mathrm{faut}$ déduire $430 \mathrm{~F}$ pour frais vétérinaires et d'aménagements. Pour obtenir une amélioration du revenu de l'éleveur, les concentrés devront donc coûter nettement moins de $420 \mathrm{~F}$ pour $100 \mathrm{UF}$, soit $4 \mathrm{~F}$ par UF. Les frais de transport des lieux de production à l'exploitation ne sont pas négligeables vu la dispersion des complexes de production et l'état actuel de l'infrastructure routière. Un prix moyen de $1 \mathrm{~F}$ par UF peut donc être raisonnablement décompté. Fabriquer des concentrés revenant à moins de $3 \mathrm{~F}$ par UF s'avère impossible pour satisfaire les besoins des animaux en période d'afflux de lait et de croissance maximale. A ce niveau, seules la mélasse de canne ( $3 \mathrm{~F}$ par UF) et les drèches fraîches (gratuites au départ brasserie) pourraient être utilisées. Le maïs (19 F l'UF) pourrait à la rigueur servir en très faibles quantités pour les brebis au premier mois de lactation.

\section{Conclusion}

Dans les conditions actuelles du marché des matières premières, des potentialités de la race Djallonké et du prix de vente des moutons, il s'avère impossible de proposer à la vulgarisation l'Unité Zootechnique améliorée telle que nous l'avons définie. Les voies à rechercher pour améliorer l'élevage du mouton au SudCameroun devront se contenter de performances intermédiaires entre celles enregistrées en milieu traditionnel et celles obtenues en station.

\section{AVENIR DE L'ELEVAGE OVIN AU CAMEROUN}

\section{Les problèmes sanitaires et leurs solutions}

Malgré la rusticité de cette race qui n'a fait l'objet d'aucune sélection, on est étonné de constater un taux de mortalité si élevé chez les jeunes. Les problèmes se multiplient dès que l'on s'adresse à des troupeaux de plusieurs dizaines de têtes. Aussi bien dans notre élevage que dans les quelques rares troupeaux importants constitués par des expatriés, nous avons constaté qu'il ne fallait pas dépasser l'effectif de 150 moutons, soit environ 60 brebis, sous peine d'avoir des problèmes sanitaires importants à résoudre et de voir la productivité par mère diminuer très fortement, même si les bâtiments sont suffisamment vastes. De même, l'introduction en quantité importante de nouveaux 
reproducteurs accroît fortement la mortalité pendant l'année qui suit. Ces problèmes généraux de gestion sanitaire étant réglés, l'éleveur d'un troupeau se verra confronté aux maladies suivantes :

\section{Boiteries et piétin}

Pendant longtemps, ce problème nous a préoccupé. Malgré l'utilisation d'un pédiluve dans lequel nous avons essayé quelque 7 produits différents, il n'est pas rare qu'en saison des pluies $1 / 4 \mathrm{du}$ troupeau soit en traitement au lazaret. Nous avons essayé un certain nombre de formules jusqu'aux piqûres intraveineuses de Bismuth. La situation ne s'améliorait pas de façon durable. Finalement nous avons pratiqué la sélection en réformant, en 1970, tous les reproducteurs fréquemment atteints. Les quelques animaux encore atteints sont isolés sur litière bien sèche. Leurs pattes sont nettoyées, désinfectées et pulvérisées à l'aide d'un Spray antiseptique. Depuis, les cas de boiteries sont devenus très exceptionnels bien que le pédiluve ne soit plus utilisé. La qualité de la litière nous paraît en ce domaine très importante. Une couche de $10 \mathrm{~cm}$ de copeaux de bois changée deux fois par semaine fait l'affaire si on ne dispose pas de parches de café qui constituent la litière idéale puisqu'elle ne doit être changée que mensuellement.

\section{Maladies respiratoires}

Le tableau des mortalités de jeunes a clairement mis en évidence que ces maladies : bronchites, pneumonies, etc., étaient responsables de plus de 40 p. 100 de la mortalité totale. La répartition par période de l'année montre une forte poussée durant le premier trimestre. Naturellement, on est tenté d'incriminer les forts écarts de température observés pendant la saison sèche mais une étude plus précise montre que ce facteur n'est pas seul en cause. Les facteurs humains ne sont pas négligeables en cette période suivant de près les fêtes de fin d'année : la surveillance sanitaire se relâche, et de plus les jeunes agneaux sont prédisposés du fait d'une alimentation déficiente : le fourrage devenant plus rare, les rations s'amenuisent sérieusement avec des ouvriers travaillant à l'heure et non pas au rendement. L'ensemble de tous ces facteurs affaiblit les défenses des agneaux contre le microbisme de bergerie, très important dans notre station à cause du nombre de spéculations pratiquées et de la fréquence des visiteurs venus de tous horizons.
Bien que ces maladies constituent notre principal souci, nous pensons qu'en troupeau isolé et bien géré des points de vue sanitaire et alimentaire, l'influence de ce microbisme pourrait être ramené à des proportions plus normales. Dans ce sens, nous préconisons l'administration d'un vaccin polyvalent aux âges de $4-6$ et 20 semaines et, surtout, un rappel annuel à tous les reproducteurs.

\section{Entérotoxémies et toxémies de gestation}

La part de ces toxicoses dans la mortalité des reproducteurs n'est pas négligeable : mis à part les accidents de manipulations et les renversements de vagin ( 1 par an) nous pouvons affirmer qu'elles représentent pratiquement la seule cause de mortalité dans cette classe d'âge. Dans tous les cas, les toxicoses s'attaquent aux animaux les plus productifs: brebis fécondes et béliers très précoces. L'administration de vaccin polyvalent apparaît très efficace contre les entérotoxémies si on sait éviter les erreurs d'alimentation et surtout les changements brusques de régime. La toxémie de gestation est difficile à combattre surtout avec le rythme intensif du cycle reproductif que peuvent suivre certaines brebis.

Dans tous les cas, la mortalité globale des adultes ne dépasse pas 4 p. 100 bien que certaines brebis soient conservées jusqu'à 11 agnelages et plus.

\section{Parasitoses gastro-intestinales}

\section{Strongyloses}

Nous avons essayé de les combattre par le Thibenzole puis par le Tétramisole avec des résultats intéressants au début mais, après quelques mois, les réinfestations se rapprochaient de plus en plus.

En 1970, nous avons remis en vigueur le déparasitage continu par incorporation de Phénothiazine $(10$ p. 100$)$ dans le concentré minéral qui reste en permanence à la disposition des animaux. Depuis cette date, il n'a plus fallu intervenir contre cette parasitose extrêmement meurtrière.

\section{Monieziose - (taeniasis)}

Elle affecte principalement les animaux pendant leur première année. L'hôte intermédiaire, un Oribate, étant actuellement impossible à combattre, nous traitons systématiquement tous 
les agneaux toutes les 6 semaines à partir de l'âge de un mois et demi au Mansonil-Bayer avec succès.

\section{Hémoparasites}

Nous n'avons jamais diagnostiqué un seul cas de trypanosomiase ni de piroplasmose alors que tiques, glossines et tabanidés ne sont pas rares dans notre station. Ceci confirme l'opinion couramment admise que les petits Ruminants d'Afrique Centrale sont naturellement résistants à ces maladies.

\section{Parasitoses externes}

Un passage au bain détiqueur tous les deux mois suffit dans notre station à prévenir les attaques des tiques. Les autres Acariens jouent un rôle négligeable. Par prudence, nous conseillons toutefois le passage au bain mensuellement.

\section{Ecthyma contagieux}

Cette infection apparaît sporadiquenent en station mais semble assez généralisée en brousse. Il faut cependant signaler que les complications sont rares. Les paysans le traitent en frictionnant les croûtes avec des herbes locales. En station, nous prévenons les complications chez les animaux fortement atteints par l'injection d'antibiotiques.

\section{Amélioration des structures de l'élevage ovin}

La promotion de ce secteur de production économique se heurte aux contraintes que nous avons explicitées ci-dessus et qu'il nous faut rappeler brièvement :

\section{Taille réduite des unités de production}

- 2 à 3 animaux par exploitation;

_. commercialisation faible : «élevage tirelire $»$;

-. surfaces fourragères peu importantes dans beaucoup de régions du Sud-Cameroun;

- modifications des habitudes à cause des dégâts causés aux cultures vivrières déficitaires.

Faible potentiel de la race Djallonké

- faible efficacité alimentaire: $6 \mathrm{UF} / \mathrm{kg}$ de gain;

- mortalité élevée;

— prolificité faible: 1,15 agneau par mise bas;
— rendement à l'abattage médiocre: 43 p. 100 .

Marché peu important pour la viande de mouton

- viande de luxe : deux fois plus onéreuse que celle de bœuf;

- moins appréciée que la viande de cabri (chevreau), sauf par les Musulmans;

- autoconsommation rare : un mouton abattu représente de la viande pour plus de 10 repas alors que les planteurs ne mangent qu'épisodiquement de la viande.

Cet ensemble de contraintes pose des problèmes sérieux à la vulgarisation en milieu villageois. Comment peut-on envisager l'avenit?

\section{Amélioration en milieu villageois}

Devant l'accumulation des palabres provoquées par les dégâts causés aux cultures vivrières, la divagation semble condamnée à plus ou moins court terme, au moins en saison de culture; l'accroissement de la population avec la réduction corrélative de la taille des parcelles individuelles et du temps de jachère laissant de moins en moins de pâturages exploitables.

De plus, cette situation oblige les paysans à aller cultiver de plus en plus loin de leur case; il n'est pas concevable d'utiliser rationnellement les jachères éloignées des villages. Cela supposerait d'affecter un berger à la surveillance des moutons alors que la main-d'œuvre manque souvent en saison de plantation.

Conservation de la taille des unités individuelles de production

Chaque famille disposant donc de quelques têtes, on pourrait envisager de les élever selon la méthode du "mouton de case "; les animaux étant enfermés ou parqués, le propriétaire leur apporterait fourrage et déchets de cuisine et de cultures. Cette technique, quoique pratiquée par certaines populations du Nord-Cameroun (les Kirdis) pour le bœuf, n'existe pas, à notre connaissance, pour les moutons. Par contre, dans tout le Sud et singulièrement dans la région Ouest, les porcs sont élevés suivant cette méthode qui leur convient très bien du fait de leur régime omnivore. On conçoit difficilement que le mouton puisse supplanter le porc dans cette pratique; les paysans ayant une truie en obtiendront chaque année environ deux porcs qui seront commercialisés pour quelque 10 à 
12000 F CFA contre $2500 \mathrm{~F}$ par brebis. Certes, les ovins auront surtout besoin de fourrages et les déchets ne forment qu'un complément mais l'expérience que nous avons tentée en ce sens s'est heurtée à la difficulté de modification du comportement des villageois. Le mouton va normalement chercher sa nourriture; le paysan, même de bonne volonté, conçoit mal qu'il faille apporter herbe et eau chaque jour à ses moutons. Même s'il leur apporte de l'herbe, c'est toujours en quantité insuffisante; quant à l'eau, c'est probablement le plus grave problème à résoudre surtout en période de lactation.

\section{Création de troupeaux villageois}

Regrouper tous les moutons d'un même village et gérer ce troupeau est-il techniquement possible? Mettons de côté les problèmes d'entente entre les différents propriétaires qui risquent pourtant de n'être pas faciles à résoudre. Dans chaque village ou quartier, on peut tabler sur 30 à 60 moutons, soit de 12 à $25 \mathrm{UZ}$ extensives. Un enclos et une bergerie rudimentaires devront être construits. Ce troupeau sera sous la conduite d'un berger qui veillera à le faire pâturer sur les jachères. Cette organisation permettra un début de sélection par castration de tous les mâles de faible valeur. Quant à l'alimentation, nous proposons de conserver les mères en bergerie pendant leur premier mois de lactation et de leur donner, en plus des fourrages, des déchets de cuisine; chaque propriétaire étant tenu de fournir chaque année une certaine quantité de résidus. La mise en disponibilité permanente d'un concentré minéral contenant de la phénothiazine devrait permettre d'améliorer sensiblement la productivité. Le berger sera presque occupé à plein temps et le problème de sa rémunération se pose. Ses cultures vivrières autoconsommées mises à part, un petit planteur dispose d'un revenu monétaire annuel de quelque $20000 \mathrm{~F} \mathrm{CFA.}$

La famille du berger pouvant s'occuper des cultures vivrières, ce dernier devrait recevoir comme salaire un minimum de 2500 à $3000 \mathrm{~F}$ par mois s'il assure lui-même les constructions et l'approvisionnement en concentrés minéraux. Un prélèvement de 30 à $35000 \mathrm{~F}$ devra donc être opéré sur les recettes annuelles du troupeau.

Quels avantages pourrait en tirer un propriétaire? La sélection des reproducteurs, voire l'achat d'un bélier améliorateur comme ceux proposés par notre station (50 à $60 \mathrm{~kg}$ ) permettra un gain de conformation des animaux qui pourraient peut-être être vendus autour de 3500 à $4000 \mathrm{~F}$, comme cela se voit déjà en brousse; soit un supplément de revenu par UZ de 350 à $600 \mathrm{~F}$. Cette technique serait donc valable pour des troupeaux villageois d'au moins $55 \mathrm{UZ}$; ce qui est très rare et risque de poser des problèmes sanitaires nouveaux.

A notre connaissance, ce type d'organisation a été essayé 3 fois par des missions : opérations qui se sont toutes soldées par un échec malgré l'apport de capitaux extérieurs pour créer les infrastructures.

\section{Peut-on encourager la création d'élevages de moutons?}

Ce qui vient d'être démontré ci-dessus s'applique aussi à la constitution de fermes d'élevages de moutons; dans le Sud-Cameroun peu de régions se prêtent à la création d'élevages : les terres sont déjà très occupées. L'accroissement de la population s'il n'est pas compensé par un accroissement au moins aussi important des rendements mobilise de plus en plus les jachères. Il existe cependant à l'est du pays de vastes étendues de savanes sous-peuplées $(2,6$ $\mathrm{hab} / \mathrm{km}^{2}$ ) qui pourraient avoir une vocation pastorale bien qu'elles soient situées loin des grands centres de consommation. La mise en service récente du chemin de fer transcamerounais permet d'ores et déjà de désenclaver cette zone encore très sous-exploitée. De même, le Centre (Adamaoua) et son prolongement naturel : le Grassfield de la province du NordOuest qui sont déjà des zones d'élevage bovin pourraient devenir aussi des centres de production ovine. Nous avons démontré que l'élevage ovin donnait un revenu annuel de 17000 F CFA à l'hectare alors que dans ces mêmes régions l'élevage bovin n'offre qu'un revenu de $2000 \mathrm{~F}$ par hectare. On pourrait donc s'étonner du peu d'engouement des éleveurs de ces régions pour la spéculation ovine, d'autant que la majorité d'entr'eux est islamisée, donc friande de viande de mouton. C'est compter sans les coutumes locales: traditionnellement, on mesure l'influence et la notoriété d'un personnage au nombre de bêtes à cornes qu'il possède. Peu à peu. l'économie de marché fait des percées dans ce milieu et il n'est pas aberrant de penser que l'élevage ovin y trouvera une place plus importante. Dans le cadre d'une politique nationale d'approvisionnement en viande, il est cependant 
naturel d'encourager l'élevage bovin dont la viande coûte deux fois moins cher au consommateur camerounais déjà carencé en protéines animales. Dans un cas comme dans l'autre, les problèmes de propriété foncière devront être résolus pour permettre aux éleveurs d'investir dans l'amélioration de leurs pâturages.

\section{Association moutons-cultures industrielles}

Parmi les cultures industrielles pratiquées au Cameroun, les caféières et palmeraies offrent une strate de couverture herbacée exploitable par les ovins. Si nous avons une expérience dans le domaine de l'association moutons-caféiers, les palmeraies sont de création trop récente au Cameroun pour que nous ayons pu y faire des études précises; dans ce dernier cas, nous nous référerons à des travaux analogues menés en Côte-d'Ivoire avec des taurins de race N'dama.

\section{Association moutons-caféiers}

De par sa diversité géographique, climatique et écologique, le Cameroun peut exploiter les deux types de caféiers: le Robusta en dessous de $1000 \mathrm{~m}$ d'altitude et l'Arabica sur les hautsplateaux de l'Ouest. Dans les deux cas, de nombreuses plantations industrielles, généralement mises en place par des expatriés, ont associé un troupeau de moutons à leurs caféières. C'est ainsi que nous avons pu suivre plusieurs troupeaux de 200 à 900 têtes.

La plante de couverture du Robusta est généralement le Pueraria javanica ou Kudzu, légumineuse qui constitue un bon fourrage, bien appété lorsque les animaux y sont habitués. Les performances atteintes sont celles que nous avons décrites pour l'extensif; à savoir un gain moyen journalier de l'ordre de 50 grammes. Mais il n'est pas rare de trouver des animaux de caféière dépassant les 65 grammes par jour. Une simple sélection massale sur la vitesse de croissance permettrait en quelques générations d'amener le croît moyen du troupeau autour de 60-65 grammes par jour, permettant ainsi un taux de commercialisation plus élevé. Quoi qu'il en soit, actuellement, une recette de $2500 \mathrm{~F}$ par Unité Zootechnique peut être retenue. A raison d'au moins 3 UZ extensives par hectare de caféière, le supplément de revenu à l'hectare sera de $7500 \mathrm{~F}$ par an. Une plantation de 100 hectares pourra donc accroître ses recettes de $750000 \mathrm{~F}$ par an desquels il faut déduire l'amortissement des investissements: $600 \mathrm{~m}^{2}$ couverts, achat de concentrés minéraux et paie d'un berger; le tout pour quelque $250000 \mathrm{~F}$ par an. Le café fournit les parches qui, nous l'avons signalé en son temps, constituent une litière idéale. Sans avoir de chiffres très précis, nous pouvons toutefois estimer à un minimum de $350000 \mathrm{~F}$ CFA les bénéfices apportés théoriquement à un propriétaire de 100 hectares de caféières Robusta. Nous disons théoriquement car, dans les faits, tous les éleveurs se plaignent de nombreuses disparitions: il est vrai que la surveillance est difficile à organiser lorsque les moutons sont laissés en liberté dans les caféières.

En altitude, les caféières Arabica sont couvertes de Pennisetum clandestinum ou Kikuyu, qui constitue aussi un excellent fourrage très résistant au pâturage. Nous n'avons pas de chiffres précis sur les croissances obtenues mais nous avons tout lieu de croire que celles-ci sont de même ordre de grandeur qu'avec du Pueraria. Dans ces conditions, le chiffre minimal de $3500 \mathrm{~F}$ à l'hectare de bénéfice net peut être retenu.

Nous avons fait un essai d'ensilage de pulpes de café avec des graminées qui s'est révélé intéressant: les animaux consomment bien ce produit qui représente un bon appoint de saison sèche.

Dans tous les cas, nous n'avons pas tenu compte du gain de main-d'œuvre réalisé grâce à cette technique. Les planteurs eux-mêmes, s'ils le reconnaissent volontiers, ne sont pas à même de le chiffrer. Cette économie est certainement de plusieurs milliers de francs à l'hectare et par an.

\section{Association moutons-palmeraies}

Si les moutons sont les seuls Ruminants à pouvoir exploiter la strate de couverture sans endommager les caféiers, dans les palmeraies, l'élevage bovin est possible comme l'a démontré une étude en Côte-d'Ivoire. Là aussi, le Pueraria est la plante de couverture et si nous envisageons de l'exploiter par des ovins nous devrons nous attendre à des performances et des résultats financiers analogues à ceux obtenus sous caféières, soit $7500 \mathrm{~F}$ à l'hectare.

Avec des bovins trypanotolérants comme le N'dama, on peut escompter un revenu brut de $2000 \mathrm{~F}$ à l'hectare; dans les deux cas, les économies de main-d'œuvre n'ont pas été comptabilisées mais elles représenteraient près de 50 p. 100 . Le coût des infrastructures sera pro- 
bablement plus élevé en élevage ovin mais pas au point d'inverser l'ordre des rentabilités. L'elevage ovin sous palmiers serait au minimum trois fois plus rentable que l'élevage de bovins trypanotolérants.

Dans ce qui précède, nous n'avons envisagé une exploitation de la strate herbacée de couverture qu'avec la race pure Djallonké. Il semble évident qu'avec un croisement industriel les résultats financiers seraient nettement plus intéressants, comme nous le verrons plus bas.

\section{Ateliers de production d'agneaux gris}

A très court terme, nous pouvons envisager la création, près des sources de sous-produits agro-industriels, d'ateliers de production d'agneaux gris. Cette spéculation est rendue possible par la sélection que nous avons opérée en vue d'accroître l'efficacité alimentaire de la race Djallonké. Mais il est évident que ces ateliers seraient encore plus rentables en utilisant le croisement industriel avec une race étrangère de type "viande " comme les Southdown, Ile de France...

Même en race pure, nous obtenons déjà chez 20 p. 100 de nos agneaux des gains moyens journaliers de 180 grammes entre 0 et 30 jours et de plus 100 grammes entre 30 et 150 jours. A ce rythme de croissance. les agneaux sont commercialisables à $20-22 \mathrm{~kg}$ avant 6 , voire 5 mois. Cette viande de première qualité, reconnue comme telle par tous nos clients, pourrait être vendue aux boucheries de luxe autour de $250 \mathrm{~F}$ le $\mathrm{kg}$ vif.

Les aliments de base seront, en complément des fourrages, la mélasse de canne, les drèches fraîches de brasseries et, à un moindre taux, les drèches desséchées et le maïs. Les drèches fraîches se conservant mal et n'étant actuellement disponibles qu'à Yaoundé $(100 \mathrm{~km}$ de la zone préconisée), elles seront ensilées avec des graminées préfanées. Cet ensilage constituera la base de la ration en saisons défavorables.

Nos calculs, confirmés par ceux du Centre de Recherches Zootechniques de BouakéMinankro (Côte-d'Ivoire) font ressortir à 1,8 F l'UF utilisable de Stylosanthes gracilis cultivé. Les besoins annuels de l'UZ améliorée sont, rappelons-le, de :

- 305 UF d'excellents fourrages soit $550 \mathrm{~F}$;

- 100 UF de concentré à $10 \mathrm{~F}$ soit $1000 \mathrm{~F}$; soit un coût alimentaire annuel de $1550 \mathrm{~F}$ par UZ.

A cela s'ajoutent $3000 \mathrm{~F}$ par UZ de constructions amortissables sur dix ans, soit $300 \mathrm{~F}$ l'an et $500 \mathrm{~F}$ pour frais vétérinaires et suppléments minéraux.

La taille maximale d'un troupeau a été évaluée à $70 \mathrm{UZ}$ qui rapporteraient donc près de $200000 \mathrm{~F}$ par an pour une superficie de 5 hectares environ.

On peut raisonnablement estimer qu'un berger ( $8000 \mathrm{~F}$ par mois en zone III) puisse s'occuper de deux troupeaux de ce genre. Un propriétaire de 10 hectares pourrait donc, s'il pratiquait cette spéculation, recevoir un bénéfice annuel de 300000 F CFA, soit $30000 \mathrm{~F}$ à l'hectare, ce qui dépasse le rapport à l'hectare des cultures industrielles traditionnelles mais qui, bien sûr, exige une technicité certaine du berger.

L'investissement de départ se monte à 1,5 million pour achat des brebis sélectionnées, constructions et aménagements des pâturages; soit un revenu annuel du capital de 20 p. 100.

Cette spéculation : l'agneau gris, paraît donc possible avec la race pure Djallonké. A fortiori, la pratique du croisement industriel serait-elle rentable.

Le marché des grandes villes peut être estimé à 3000 agneaux de qualité par an. On pourrait donc promouvoir dans le Sud-Cameroun des unités de l'ordre de 50 troupeaux de 70 mères.

\section{Création d'une nouvelle race}

L'accélération inéluctable de l'urbanisation et l'accroissement du niveau de vie accroîtront sensiblement la demande en viande de mouton dans les prochaines décennies. Au niveau de la recherche, il est grand temps de préparer ces solutions d'avenir.

Nous venons de démontrer longuement que la race Djallonké n'offrait pas des rendements suffisants pour la création d'élevages rentables d'ovins si le prix de la viande de mouton n'augmente pas considérablement. On peut raisonnablement penser que des paysans ne pourront créer et vivre d'élevages de moutons que si le revenu monétaire est multiplié par deux. Doubler le prix de la viande n'apparaît pas comme une solution normale en pays pauvre. Reste 
alors à essayer de doubler la productivité de l'UZ extensive.

La race locale Djallonké présente de bons atouts quant à ses qualités maternelles; on peut lui reprocher principalement une prolificité faible et une valeur laitière médiocre. Sa principale qualité réside dans son rythme d'agnelage qui, nous l'avons démontré, peut être encore amélioré par sélection. De plus, elle résiste particulièrement bien aux conditions pathologiques de la zone forestière: trypanosomiase, piroplasmose, dermatoses...

Le principal verrou à faire sauter est la faible productivité de 1'UZ liée à la prolificité modeste et à la forte mortalité des jeunes. Le second point peut être amélioré mais n'apportera pas la rentabilité suffisante permettant de créer des élevages ovins.

Nous pensons donc qu'une " nouvelle race locale " doit être créée alliant les qualités de la race Djallonké à la prolificité et à la valeur laitière de races réputées rustiques et prolifiques comme les races Romanov et Finnoise. L'une et l'autre sont très résistantes aux conditions difficiles de milieu et mettent bas autour de 3 agneaux par portée. La création d'une race demi-sang Djallonké-Romanov serait probablement assez rustique et aurait une prolificité minimale de 200 .

Dans ces conditions, l'UZ construite sur cette race pourrait commercialiser chaque année autour de 1,95 agneaux, plutôt mieux conformés que les animaux locaux. Le revenu annuel par UZ serait done de $8000 \mathrm{~F}$ pour des dépenses de l'ordre de $2800 \mathrm{~F}$ (conditions amé- liorées) soit un bénéfice de $5200 \mathrm{~F}$ par UZ et de $365000 \mathrm{~F}$ par troupeau de 70 mères nécessitant 7 hectares. Un revenu annuel de plus de $50000 \mathrm{~F}$ à l'hectare pourrait devenir une affaire très intéressante.

Ce profit pourrait encore être considérablement accru par la pratique du croisement industriel entre ces mères “Djallonké prolifiques ") et une race à viande. Dans tous les pays du monde, l'élevage du mouton s'oriente vers la stratification et le croisement à plusieurs étages. C'est également pour le Cameroun la seule voie pour tirer profit des qualités de la race locale et de ses pâturages guinéens abondants situés en zone trypanosomées. Dans cette perspective, la création d'une station de création, de sélection et de diffusion de cette nouvelle race s'avère indispensable dans les plus brefs délais.

\section{REMERCIEMENTS}

Les auteurs tiennent à remercier tous les collaborateurs qui les ont aidés durant ces huit années de recherches: Dr DUMEZ, Dr BOTTON, G. MARTET, R. GORDON et tous les bergers Camerounais. Notre gratitude va également aux éleveurs qui ont mis leurs troupeaux à notre disposition pour y effectuer nos relevés; en particulier les sociétés CFSO d'AbongMbang et d'Atock, SINCOA de Babadjou, mission de Minta, ZAPI de Menguémé. Enfin, nous ne saurions terminer sans remercier tous les étudiants de $1^{\prime} E N S A$ de la $6^{\mathrm{e}}$ à la $10^{\mathrm{e}}$ promotion qui ont mené les enquêtes villageoises lors de leurs "stages de monographies villageoises ").

\section{SUMMARY}

Djallonke sheep breed in Cameroon

Zootechnical possibilities, breeding conditions, future

The authors have been following for nine years the study and the selection of the Djallonke sheep, both in the station of Nkolbisson, located $10 \mathrm{~km}$ from Yaounde (Cameroon) and through multiple surveys conducted in several areas of Western and Central Cameroon. Data and results obtained in the zootechnical ant veterinary as well in the socio-economic fields, let foresee the interesting possibilities for improvement of this breed. Nevertheless, according to the actual economic context, intensive production of Djallonke sheep is not very profitable. Further more, milk production of the ewes would have to be increased. For this purpose, some exotic blood coming from prolific and good milker breeds - Romanov for example - could be infused. 


\section{RESUMEN}

La raza ovina Djalonke en Camerún

Potencialidades zootecnicas, condiciones de cria, porvenir

Los autores han estudiado durante nueve años la selección de la oveja Djalonke en la explotación de. Nkolbisson situada a $10 \mathrm{~km}$ de Yaunde, Camerún y en numerosas regiones del oeste y del centro de Camerún. Las informaciones y los resultados obtenidos, desde el punto de vista zootecnico, veterinario y socio-económico muestran las importantes posibilidades de mejora de dicha raza - Sin embargo, con las condiciones económicas actuales, la cria intensiva de la oveja Djalonke no se comproba interesante. Necesitaria mejorar las posibilidades lecheras de las ovejas, lo que podria realizarse por el cruzamiento con una raza prolifica y lechera como la raza Romanov.

\section{BIBLIOGRAPHIE}

1. Approvisionnement en viande de l'Afrique centrale (2 tomes). Paris, S.E.D.E.S., 1971.

2. DOUTRESSOULLE. L'élevage en Afrique occidentale française. Paris, Maisonneuve et Larose, 1947.

3. BELSCHNER (M. G.). Sheep management and diseases. 8th ed., Melbourne, Angus et Robertson.

4. BRANCKAERT (R.). Nécessité d'une étude physio-zootechnique approfondie des petits ruminants en Afrique centrale. 2e Conférence Mondiale sur la production animale, Université de Maryland (U.S.A.), 1968.

5. BRANCKAERT (R.), VALLERAND (F.). Production de viande à partir des petits ruminants en Afrique centrale. Colloque O.C.A.M. sur l'Elevage, Fort-Lamy, Tchad, 1969.

6. CURASSON (G.). Le mouton au Soudan français. Paris, L'Union ovine coloniale, s.d.
7. DERAMEE (O.). L'élevage du mouton en Afrique centrale. CEDESA, 42, rue du Commerce, Bruxelles-4, 1967.

8. FALCONNER (D.S.). Introduction to quantitative genetics. Edimburgh, Oliver and Boyd.

9. LEROY (A. M.). Le mouton. Paris, Hachette, 1968.

10. Rapports d'Activités du Centre de Recherches Zootechniques de Sotuba (Mali), 1953 à 1957.

11. Rapports de stage d'analyse du milieu des étudiants de l'E.N.S.A., Yaoundé, Direction des Etudes. Ecole Nationale Supérieure Agronomique.

12. ROMBAUT (D.). Elevage bovin sous palmiers. Abidjan, Ministère de la production animale, 1972.

13. VALLERAND (F.). Génétique de la taille de portée et possibilités de sélection sur la prolificité chez différentes espèces domestiques. Mémoire DEA-FAC. Sci., Paris, 1967. 